\title{
EL DESARROLLO DE LA RAZÓN PRÁCTICA EN ESTUDIANTES UNIVERSITARIAS DEL AMBITO DE LA SALUD, SEGÚN EL ENFOQUE DE MARTHA NUSSBAUM
}

\author{
THE DEVELOPMENT OF PRACTICAL REASONING IN UNIVERSITY STUDENTS IN THE AREA \\ OF HEALTH, ACCORDING TO MARTHA NUSSBAUM'S APPROACH \\ O DESENVOLVIMENTO DO RACIOCÍNIO PRÁTICO NOS ESTUDANTES UNIVERSITÁRIOS NO \\ DOMÍNIO DA SAÚDE, DE ACORDO COM A ABORDAGEM DE MARTHA NUSSBAUM
}

\author{
Paula Oyarzún Andrades ${ }^{1}$, Francisco Esteban Bara ${ }^{2}$, Teodor Mellen Vinagre ${ }^{3}$ \\ ${ }^{1}$ Universidad de Valparaíso, Chile, paula.oyarzun@uv.cl \\ ${ }^{2}$ Universidad de Barcelona, España, franciscoesteban@ub.edu \\ ${ }^{3}$ Universidad ESADE, España, teodor.mellen@esade.edu
}

Fecha de recepción: 31 agosto 2020

Fecha de aceptación: 28 septiembre 2020

\section{RESUMEN}

Por siglos el estudio de las capacidades humanas ha despertado gran interés entre educadores(as), filósofos(as) y pensadores(as) en torno al cultivo del ser humano. La filósofa Martha Nussbaum desarrolló el Enfoque de las Capacidades (EDC) en cuyo eje reconoce la razón práctica como herramienta para contrarrestar las inequidades de género y avanzar en materia de justicia social. El objetivo de la investigación fue comprender la percepción sobre el desarrollo de la razón práctica, en estudiantes mujeres que cursaron cuarto año de la carrera de Obstetricia y Puericultura de la Universidad de Valparaíso (Chile) en el período 2017-2018, a partir de sus experiencias asociadas con el currículo y vida universitaria. El enfoque fue hermenéutico-fenomenológico de carácter cualitativo, a través de entrevistas en profundidad. Los resultados develaron información significativa sobre la potenciación del pensamiento crítico, la imaginación creativa, la expresion de sentimientos y la libertad de conciencia, a través de diversas experiencias formativas, otorgando oportunidades para acrecentarlas y consolidarlas a lo largo de sus vidas, a partir de prácticas sociales que promueven el respeto por el otro desde una mirada inclusiva, ética y biopsicosocial.

Palabras Claves: Capacidades humanas; razón práctica; educación superior; enfoque de capacidades.

\section{ABSTRACT}

For centuries the study of human capabilities has aroused great interest among educators, philosophers and thinkers in the cultivation of human beings. The philosopher Martha Nussbaum developed the Capabilities Approach (CA), which recognizes practical reason as a tool to counteract gender inequities and advance social justice. The objective of the research was to understand the perception of the development of practical reason in female students who are in their fourth year of the Obstetrics and Childcare career at the University of Valparaiso (Chile) in the period 20172018, based on their experiences associated with the curriculum and university life. The approach was qualitative hermeneutic-phenomenological, through in-depth interviews. The results revealed significant information about the empowerment of critical thinking, creative imagination, expression of feelings and freedom of conscience,

1 El desarrollo de la razón práctica en estudiantes universitarias del ámbito de la salud, según el enfoque de Martha Nussbaum 
through various formative experiences, providing opportunities to increase and consolidate them throughout their lives, from social practices that promote respect for others from an inclusive perspective, ethical and biopsychosocial.

Key words: human capabilities; practical reason; higher education; capability approach

\section{RESUMO}

Por séculos o estudo dascapacidadeshumanas há despertadograndeinteresseentreeducadores, filósofosepensadores em torno ao desenvolvimento do ser humano. A filósofa Martha Nussbaum desenvolveu o Enfoque das Capacidades (EDC) em cuja base reconhece a razão prática como ferramenta para contra restar as inequidades de gênero e avançar em matéria de justiça social. O objetivo da investigação foi compreender a percepção sobre o desenvolvimento da razão prática, em estudantes do gênero feminino que cursaram o quarto ano da carreira de Obstetrícia e Puericultura da Universidade de Valparaíso (Chile) no período 2017-2018, a partir de suas experiências associadas com seu currículo e vida universitária. $\mathrm{O}$ enfoque foi hermenêutico - fenomenológico de carácter qualitativo, a través de entrevistas em profundidade. Os resultados revelaram informação significativa sobre a capacitação do pensamento crítico, imaginação criativa, expressão de sentimentos e liberdade de consciência, através de várias experiências formativas, proporcionando oportunidades para as aumentar e consolidar ao longo das suas vidas, com base em práticas sociais que promovem o respeito pelos outros numa perspectiva inclusiva, ética e biopsicossocial.

Palavras chaves: Capacidades humanas; razão prática; educação superior; enfoque de capacidades

\section{1.- INTRODUCCIÓN}

Los avances de las últimas décadas en Educación Superior relevan en sus proyectos la necesidad de considerar al estudiante y sus aprendizajes como actores principales durante el proceso formativo, para que logre ser capaz de cultivar todas sus capacidades a lo largo de su vida. La filósofa estadounidense Martha C. Nussbaum, al principio de la década de los ochenta, se adentra en el estudio de los planteamientos aristotélicos sobre el desarrollo de la imaginación creativa, la sabiduría práctica, la importancia de lograr la justicia social y promover la formación de las personas, planteando el Enfoque de las Capacidades (en adelante EDC).

Este nuevo paradigma teórico presenta una naturaleza interdisciplinar que ha tenido gran influencia en el campo del desarrollo económico, político, social y cultural, movilizando políticas públicas de diversas naciones en el mundo. Esta propuesta se aleja del método tradicional utilitarista que mide la calidad de vida de las personas a partir de la medición del PIB (producto interno bruto) de cada nación, destacando la impor- tancia de comprender y analizar el contexto narrativo de las vidas humanas. De esta manera, presta especial atención a los relatos y vivencias de las personas mediante el estudio de lo que piensan y creen que pueden ser y hacer en sus vidas. Estas habilidades denominadas internas pueden ser innatas, o bien desarrolladas y estarían influenciadas o relacionadas con las oportunidades que le entrega el entorno para vivir una existencia plena y digna (Nussbaum, 2012a).

Para Nussbaum (2005), la educación desempeña una función fértil, pues abre opciones de muchas clases. Investigar posibles capacidades y funcionamientos fértiles ayuda a detectar puntos donde pueden intervenir más adecuadamente las políticas públicas de cada estado. En su libro "El cultivo de la humanidad", plantea que la educación es una actividad clave y valiosa en sí misma, capaz de generar autoestima, entendimiento mutuo y posibilidades de participación social. Para la filósofa, la Educación Superior debe tener por meta crear una comunidad de personas que desarrollen el pensamiento crítico, que busquen la verdad más allá de las barreras de clase, género y nacionalidad, que respe-

2 El desarrollo de la razón práctica en estudiantes universitarias del ámbito de la salud, según el enfoque de Martha Nussbaum 
ten la diversidad y la humanidad. En esta propuesta, la capacidad se plantea como una aptitud del individuo, la cual representa lo que una persona puede hacer, ser y lograr. Así mismo son necesidades comunes de todas los seres humanos, independiente del género, sexo, clase, raza o nacionalidad (Monereo, 2015). La filósofa plantea que el desarrollo de la capacidad de razón práctica se lograría a través del fomento en las aulas de la literatura y las artes, las cuales a su vez cultivan otrasy lograr. Así mismo son necesidades comunes de todas los seres humanos, independiente del género, sexo, clase, raza o nacionalidad (Monereo, 2015). La filósofa plantea que el desarrollo de la capacidad de razón práctica se lograría a través del fomento en las aulas de la literatura y las artes, las cuales a su vez cultivan otras capacidades intrínsecas, tales como la creatividad, la imaginación, el juicio crítico y la sensibilidad social. Para Nussbaum, considerarlas en los curriculum universitarios sería la vía mas propicia para el entendimiento general humano (Nussbaum, 2001).

En consecuencia, las razones que motivan esta investigación son retomar los planteamientos filosóficos y humanistas que reconocen al ser humano como fin en sí mismo, siendo capaz de interpretar lo que las personas necesitan para desarrollarse plenamente en la vida. Se explora sobre la percepción que tienen las estudiantes de la carrera de Obstetricia y Puericultura de la Universidad de Valparaíso, sobre el desarrollo de su razonamiento práctico en cuanto a poder formarse una concepción del bien y reflexionar críticamente acerca de la planificación de su propia vida.

Los resultados obtenidos permiten orientar la toma de decisión y las estrategias de apoyo al desarrollo humano que emanan de esta comunidad universitaria, para el mejoramiento de la calidad en los procesos formativos de profesionales del ámbito de la salud, haciendo posible que las estudiantes lleven vidas universitarias plenas y dignas. Cabe señalar que en Chile no hay estudios ni publicaciones sobre la adopción de este enfoque en educación en carreras del área de la salud. De esta manera, este estudio aporta evidencia científica sobre el rol preponderante de la Educación Superior en el desarrollo esta capacidad durante la formación profesional. Esta investigación extrae los principales resultados de la dimensión razonamiento práctico, desarrollada por la autora en su tesis doctoral en Educación y Sociedad de la Universidad de Barcelona (España).

\section{MATERIAL Y MÉTODO}

\subsection{Marco Conceptual}

La fuente occidental histórica más temprana e importante en el pensamiento ético-político de Nussbaum fue Aristóteles. La autora ha reflexionado sobre la filosofía de él a lo largo de su trayectoria profesional desde diversas áreas del desarrollo humano, entre ellas la sabiduría práctica, derivando a su propuesta central en el plano de las capacidades humanas.

Para Fonnegra (2013) en lo que respecta al desarrollo de la razón práctica señala que Nussbaum presenta una concepción amplia de ella, en la que se reivindica la importancia de la percepción de casos particulares, de las emociones y de la imaginación para juzgar diferentes alternativas que comprometen la deliberación racional. En este contexto, la lectura de narraciones literarias ocupa un lugar preponderante, puesto que quien las sigue encuentra casos ejemplares que amplían el horizonte de la formación del juicio en torno a cómo llevar a cabo una buena vida.

En el plano de la imaginación narrativa, Nussbaum (2001:118) citando a Aristóteles (siglo IV a.C) en el capítulo 9 de su Poética señala que la literatura nos muestra "no las cosas que han sucedido, sino aquellas que podrían suceder..." Tomándose de esta idea para la filósofa la literatura y las artes tales como la música, danza, pintura, escultura y la arquitectura cultivan las capacidades de la creatividad, la imaginación, el juicio crítico y la sensibilidad social. Aristóteles argumenta en este capítulo que la literatura es "más filosófica que la historia", con lo que quiere decir según Nussbaum, que es una vía más propicia para el entendimiento ge- 
neral humano. Como la literatura desempeña un papel vital en la educación orientada a formar ciudadanos del mundo, tiene sentido preguntar de qué manera puede cumplir con esta función, y de la mejor forma posible; qué tipo de obras literarias y qué formas de enseñarlas deberían fomentar las instituciones de educación para promover una visión inclusiva, informada y receptiva del otro/a, de quien es diferente a uno/a (Nussbaum, 1998).

Según Nussbaum, la inteligencia práctica no tiene que ver con el saber teórico, ésta no se adquiere huyendo de experiencias mundanas, siguiendo principios formales o maximizando el placer individual (Nussbaum, 2001). En el ámbito de la educación en salud este tema es muy relevante, debido a que se trabaja al cuidado de las personas, esto conlleva a interactuar permanentemente con individuos que poseen diversas personalidades, creencias y costumbres; de tal manera que entregar los conocimientos teóricos frente a una determinada situación no basta, se requiere actuar en virtud del conocimiento práctico que otorga la vida reflexiva, el pensamiento crítico y los principios éticos. En este sentido, la educación universitaria tiene el desafío de implementar en sus currículos instancias que potencien el desarrollo de esta capacidad, adentrando al estudiante a vivir experiencias lo más cercanas al contexto en que se desempeñará en su profesión.

Por otra parte, Nussbaum no concibe la imaginación solamente como ensueño o escape de la realidad, sino que retoma el sentido del concepto aristotélico de phantasia, el cual apunta al reconocimiento de la posibilidad que tiene el hombre y mujer de superar las particularidades del mundo interior y de su presente inmediato. La phantasia es entonces fundamental al momento de deliberar sobre un particular, esto es al momento de tomar decisiones en las que se compromete la elección de un bien en lugar de otro (Fonnegra, 2013). Es gracias a la facultad imaginativa que el ser humano puede pensar cómo alcanzar la excelencia, cómo moldear su carácter, cómo evaluar las situaciones vividas, de modo que sin ella sería imposible construir un proyecto de vida conforme a un ideal de virtud.

En suma, Nussbaum reivindica la concepción aristotélica de la sabiduría práctica y la imaginación narrativa para darle importancia a los parámetros de vida comunitaria, por reconocer las contingencias de la existencia humana, el pluralismo de los valores, la heterogeneidad de nociones del bien, el aporte de la emoción, de la imaginación y de la libertad de conciencia al momento de elegir y de buscar un ideal de vida. Con estas convicciones propone a los establecimientos de Educación Superior que repiensen el currículo y que desde los primeros años incorporen programas que fomenten las experiencias comunitarias, la lectura narrativa, las artes y las humanidades.

\subsection{Objetivo de la investigación}

Comprender las percepciones sobre el desarrollo de la capacidad de razón practica, según el enfoque de Nussbaum en estudiantes mujeres que cursan cuarto año de la carrera de Obstetricia y Puericultura de la Universidad de Valparaíso (Chile) en el periodo 20172018, a partir de sus experiencias asociadas con el currículo y vida universitaria.

\subsection{Enfoque metodológico y categoría estudiada}

El enfoque fue hermenéutico-fenomenológico de carácter cualitativo, con una clara orientación introspectivo-vivencial, a través del cual la investigadora se adentró en el mundo que rodeó a las estudiantes durante su vida en la universidad, reconociendo además sus experiencias previas, intentando comprender e interpretar los simbolismos socioculturales inmersos en ellas. Se hace preponderante el desarrollo de la metodología de investigación cualitativa, la cual es una actividad sistemática orientada a la comprensión en profundidad de fenómenos educativos y sociales, a la transformación que ellas se sintieron capaces de ser y hacer, con relación a la capacidad de razonamiento práctico, a partir de sus diversas experiencias universitarias asociadas con el currículo que cursaron. Se buscó responder a la pregunta central: ¿Cómo es percibido el desarrollo 
de su capacidad de razón práctica, según el enfoque de Martha Nussbaum, por las estudiantes de cuarto año del curso académico 2017-2018 de la carrera de O y P de la Universidad de Valparaíso y cómo valoran su presencia en el currículo y en sus vidas universitarias?. La resolución de estas y otra cuestiones motivó a la investigadora a sumergirse en el contexto sociocultural en que se desenvolvieron las estudiantes propiciando un clima dialógico y contemplativo. En este sentido, Ruiz (2011: 17) señala que la investigación cualitativa enfatiza conocer la realidad desde una perspectiva insider: "captar el significado particular que a cada hecho atribuye su propio protagonista y de contemplar estos ele- mentos como partes de un conjunto sistémico". Desde el punto de vista epistemológico, el acento de la investigación se orientó hacia la fenomenología sociológica (Schutz, 1995).

La categoría estudiada se construyó a partir de dimensiones y subdimensiones, en torno al concepto de capacidad de razón practica. Cabe señalar que esta tabla fue incorporando más subdimensiones en la medida que en el discurso de las estudiantes emergieron otras capacidades y experiencias necesarias de analizar e interpretar. A continuación, se presenta una tabla resumen con la principal dimensión y subdimensiones estudiadas:

\section{Categoría de estudio}

\begin{tabular}{|c|c|c|c|}
\hline Objetivo & Pregunta & Dimensión & Subdimensión \\
\hline \multirow[t]{4}{*}{$\begin{array}{l}\text { Capacidad Humana } \\
\text { interiorizada }\end{array}$} & $\begin{array}{l}\text { ¿Cómo desarrollo la } \\
\text { capacidad? }\end{array}$ & Razonamiento práctico & Pensamiento crítico \\
\hline & $\begin{array}{l}\text { ¿Qué me aportó la for- } \\
\text { mación universitaria? }\end{array}$ & & $\begin{array}{l}\text { Protección de libertad de } \\
\text { conciencia }\end{array}$ \\
\hline & & & $\begin{array}{l}\text { Utilizar los sentidos y la } \\
\text { imaginación }\end{array}$ \\
\hline & & & $\begin{array}{l}\text { Emocionarse y expresar } \\
\text { sentimientos }\end{array}$ \\
\hline
\end{tabular}

Tabla 1: Operacionalización de categoría

\subsection{Acceso al campo y selección de los participantes}

Para acceder al trabajo de campo se solicitó apoyo formal a las autoridades de la universidad, durante este proceso se accedió a la información fundamental en torno a los espacios físicos, sociales y académicos en que se desenvolvieron las estudiantes. Se realizó un vagabundeo de las principales instalaciones de la Facultad de Medicina, que es donde transcurrió la mayor parte de su vida universitaria. En esta etapa la investigadora convivió con las protagonistas del proceso investigati- vo asumiendo un rol investigador -participante, que le permitió crear confianza e ir impregnándose de los significados del grupo. La selección de la muestra se realizó intencionadamente, a partir de criterios de representatividad del discurso. Tomando en cuenta que la propuesta del EDC de Nussbaum centra su atención en personas de sexo femenino, con factores de vulnerabilidad y de nivel socioeconómico medio-bajo, toda vez que para la filósofa este grupo de personas presentan mayores dificultades a lo largo de sus vidas para alcan- 
zar un umbral de capacidades que les permita vivir una vida plena y digna. Por consiguiente, la muestra estuvo conformada por 12 estudiantes mujeres, con esta cantidad se alcanzó el criterio de saturación del discurso. Se efectuaron entrevistas en profundidad en un espacio fisico asignado dentro de la facultad, se procuró tener un ambiente de confianza, privacidad y comodidad con las participantes. Como señala Spradley (1979: 58) la entrevista es posible concebirla como: "una serie de conversaciones libres en las que el investigador poco a poco va introduciendo nuevos elementos que ayudan al informante a comportarse como tal".

\subsection{Técnicas de análisis}

La totalidad de las entrevistas se transcribieron posterior a su grabación, fueron leídas y analizadas minuciosamente para su posterior codificación. La categorización se basó inicialmente en el marco conceptual del EDC de Nussbaum y luego se complementó con los relatos emergentes de las estudiantes en cuanto al desarrollo de esta capacidad y su asociación con diversas subdimensiones como pensamiento critico, la libertad de conciencia, los sentidos, la imaginación y por último la expresión de emociones y sentimientos. De esta manera, se efectuó un análisis comprehensivo de las narrativas y experiencias de las estudiantes durante las entrevistas. También se realizó una triangulación subjetiva entre las vivencias y los discursos de las entrevistadas, en torno al desarrollo de la capacidad de razón práctica, el EDC y la influencia que tuvo la vida universitaria y el contexto socioeducativo en que estuvieron inmersas.

Para el análisis cualitativo se utilizó como software de apoyo el programa NVivo11 para Windows. Como recurso tecnológico la investigación se apoya del Sistema integrado de bibliotecas UV, base de datos EBSCO, Scopus y programa software gestor bibliográfico Zótero (versión 5.0.37).

\subsection{Consideraciones éticas}

Cualquier actividad de investigación en la que participen seres humanos plantea ciertos dilemas sobre lo éticamente correcto o incorrecto de determinadas actuaciones. La pluralidad de métodos utilizados en la investigación en educación, basados en diferentes perspectivas epistemológicas, hace que las consideraciones éticas posean particularidades propias dependiendo del paradigma del investigador (Tójar y Serrano, 2000: 1).

El estudio protegió desde el punto de vista ético todos los aspectos sensibles que rodearon la investigación, desde el acceso al campo mediante la realización de entrevistas con autoridades de la carrera, obtención de cartas de permiso y autorización del estudio, hasta la confidencialidad y protección de los relatos que aportaron las estudiantes durante las entrevistas, relevando tres aspectos a saber: Selección justa de los sujetos, la investigación estuvo orientada a estudiantes de sexo femenino de cuarto año, que aportaron información significativa sobre sus vivencias y percepciones; Consentimiento informado: las entrevistas se grabaron por audio previa presentación de los objetivos del estudio por parte de la investigadora, lectura y firma de consentimiento informado que dio cuenta del carácter voluntario y anónimo de éste, título del estudio, propósitos y procedimiento a desarrollar. Se planteó la libertad de retirarse cuando la estudiante lo desee, se señaló que la investigación no reviste obligatoriedad, ni coacción, ni interés pecuniario. También aportó datos asociados a la investigadora principal en caso de requerir contactarla; y respeto por los sujetos de investigación: el uso de los datos fue confidencial con cadena de custodia, es decir se asignó código alfanumérico a cada entrevista transcrita, cabe señalar que los nombres asignados fueron ficticios. Se guardaron las transcripciones y cartas de consentimiento en un mueble bajo llave a cargo de la investigadora principal. 


\subsection{Pensamiento crítico}

Las reflexiones de las estudiantes en torno al desarrollo de esta capacidad durante su formación universitaria, desde la óptica del saber ser y actuar frente a los demás, fueron múltiples y enriquecedoras para la investigación. Al adentrarse en el significado de las experiencias en las estudiantes, cursando la carrera y experimentando paulatinamente la disciplina en diversas actividades intra y extracurriculares; las reflexiones convergieron en torno a reconocer que en el interior de las aulas existió un clima de aprendizaje propicio para dialogar sobre diversos temas de interés, la mayoría destacó que los docentes propiciaron una comunicación horizontal con ellas, tanto dentro como fuera de clases. "No solamente cómo nos entregaban el conocimiento para nosotras estudiar, si no que nos hacían participar con ellos, yo creo que eso me fue cambiando el pensamiento..." (E1).

En su libro El cultivo de la humanidad (2001), Nussbaum reflexiona sobre la educación antigua y el desarrollo de la academia del pensamiento de Sócrates, en la cual predominaba el diálogo y la indagación socrática, es decir la expresión de las ideas entre el maestro y el aprendiz, la formulación de interrogantes o preguntas de alto nivel cognitivo y el cuestionamiento permanente de lo aprendido como constructo de una ciudadanía libre y democrática. En este sentido, las estudiantes creen que la carrera propendió a esta intención desde los primeros años de formación. Sin embargo, también plantearon que existió la necesidad de analizar temáticas emergentes con mayor profundidad e idealmente en contextos reales, a partir de instancias de trabajo conjunto no solo en las aulas como suele darse, sino en espacios abiertos para la comunidad estudiantil donde puedan plantearse temas de contingencia nacional y mundial, tales como el aborto terapéutico, la violencia de género, la educación no sexista, las inmigraciones, el cuidado medio ambiental, entre otros. Estas opiniones entreabren una gran oportunidad para la carrera, toda vez que demostraron un interés significativo por parte de las entrevistadas de generar espacios abiertos que faciliten el desarrollo de opiniones, contrapuntos, divergencias, consensos y debates de diversos temas que no solo atañen a la disciplina, de alguna manera se devela el interés por abrirse al mundo desde diversas visiones.

"Lo que uno quiere es que los profesores, como profesionales puedan tomar estos temas y bajarlos, pero de una manera real" (E2).

Varias estudiantes destacaron la importancia del trabajo en equipo, mediante el desarrollo de prácticas o talleres en terreno junto a las comunidades, considerando que esta vinculación con las personas y actores sociales contribuyó a un aprendizaje más significativo, profundo y transformador.

"Los talleres que tuvimos con las comunidades también nos ayudaron, porque nos hicieron salir de nuestra estructura, espacio físico y nos llevaron a otro lado, a involucrarnos con una comunidad..." (E2).

Estos planteamientos son muy importantes de abordar y de alguna manera se reflejan en las interrogantes de Nussbaum en cuanto a plantearse ¿qué tipos de ciudadanos están formando y en qué medida lo están logrando? (Nussbaum, 2001). En tal sentido, la filósofa propone que el nuevo énfasis en la diversidad de los currículos en las escuelas superiores y universidades es un modo de hacerse cargo de los nuevos requisitos de la condición de ciudadano, de los deberes, derechos y privilegios que le son propios; un intento de formar personas que puedan funcionar como ciudadanos no solo de algunas regiones o grupos locales, sino también como ciudadanos de un mundo complejo, cambiante e interconectado.

También se constató en los relatos el profundo significado y valor que le atribuyeron a la realización de sus prácticas clínicas y comunitarias durante su formación universitaria, y cómo estas experiencias contribuyeron a potenciar su pensamiento crítico, a partir del desarrollo de diversas habilidades cognitivas, procedimentales y actitudinales, que le permitieron aprender en 
contextos reales, de tal manera que puedieron empoderase de su rol, integrar saberes, comunicarse mejor y ser más reflexivas al momento de expresar una opinión y/o tomar una decisión.

"Sobre todo por las prácticas...que te hace abrir los ojos y ver distintas realidades ponerse en el lugar de otra persona, para aprender cómo atender a la gente" (E3).

Además, en su mayoría reconocieron que las prácticas les permitieron desarrollar más la personalidad, autoestima y pertenencia, toda vez que se sintieron capaces de aportar positivamente dentro del equipo de salud.

"En especial ahora que estoy en práctica en consultorio siento que me han tomado muy en cuenta que me han dejado ser parte y me siento así, aunque no soy funcionaria me siento parte del equipo..." (E4).

Según los diversos relatos aportados, se considera que durante la trayectoria universitaria se produciría un salto cualitativo, desde las experiencias propias de la adolescencia a la vida adulta, que conlleva consigo el conocimiento profundo del propio ser (vida reflexiva), asumir responsabilidades con uno mismo/a y los otros/as, convivir con experiencias diversas, construir puntos de opinión en torno a diversas problemáticas de la vida diaria y aprender haciendo en la medida que se avanza a cursos superiores. Estas vivencias revelaron que las estudiantes ad-portas de egresar se sintieron capaces de asumir su rol profesional y fueron capaces de cursar vidas de manera más reflexivas y autónomas.

\subsection{Protección de la libertad de conciencia}

Hay un punto en que convergieron la amplia mayoría de los relatos y tuvo relación con la influencia recibida desde sus núcleos familiares en torno a creencias, ideologías, posturas valóricas, diversos puntos de vista y sus formas de expresarlas y vivirlas.

"Yo me crie con mis abuelos, mi abuela es terriblemente machista...entonces ninguna posibilidad de hablar de esos temas..." (E4).

En sus narrativas hubo consenso que la universidad les cambió su forma de plantearse frente a situaciones propias del contexto sociocultural y político en que estuvieron inmersas. En este sentido, varias estudiantes reconocieron que los movimientos estudiantiles de los últimos años, en torno a diversas demandas sociales como gratuidad y fin al lucro en educación, educación para todos/as (universal) y de calidad; significaron experiencias universitarias nuevas que les permitieron salir del espacio de comodidad en el que se encontraban y generarse una opinión propia y critica de las necesidades sociales. Así mismo, muchas de las entrevistadas reconocieron que por primera vez habían participado en marchas estudiantiles, a favor de los derechos y la dignidad de las personas.

"En la universidad yo conocí un ambiente mucho más diverso..., las mismas marchas, el movimiento estudiantil... en el que trabajé mucho, daban otra perspectiva de vida..." (E5).

Esta realidad universitaria, se adhiere al EDC, pues Nussbaum propone contrarrestar a través de la educación, las inequidades ligadas al lugar de nacimiento. Su propuesta de educación cosmopolita se dirige a formar ciudadanos preocupados por sus semejantes, sin importar su ubicación geográfica ni contexto sociocultural (Nussbaum, 2012b). En la misma línea, Arjona Pachón (2013) explica que la propuesta filosófica de Nussbaum sobre la naturaleza del liberalismo político puede ser abordada desde diversos aspectos. Con respecto a la naturaleza y los fines generales de la política, su pensamiento propone un intento por recuperar la antigua pregunta por la vida buena y la forja del carácter, sin perder de vista la relevancia que siglos de pensamiento liberal han dado a la perspectiva de la justicia, cuya pertinencia se conserva en el mundo globalizado que habitamos, en el que coexisten diversas concepciones comprehensivas del bien, y las diferencias culturales se ven afectadas por problemas, oportunidades y desafíos similares. En este sentido, la experiencia durante la trayectoria universitaria y las 
demandas propias de los movimientos sociales en torno a diversas temáticas aportó positivamente en la forja del carácter de las estudiantes entrevistadas.

\subsection{Utilizar los sentidos y la imaginación}

Las reflexiones en torno a esta temática fueron más sucintas, la investigadora percibió que hay cierto grado de dificultad por parte de las estudiantes de reconocer el desarrollo de estas capacidades durante su formación universitaria. Dentro de los aspectos que destacaron en sus discursos estuvo la impronta que les entregaron algunas asignaturas de los primeros años de estudio descritas por algunas de ellas como lúdicas; sin embargo, reconocen que en cursos superiores estas actividades van desapareciendo.

"En primer año había ramos como psicoafectiva, que había que hacer hartas manualidades y cosas así, en ese sentido sí, pero... creo que después se perdió..." (E6).

En cuanto a esta capacidad, la mayoría de las estudiantes opinaron que las desarrollaron previo a sus estudios universitarios, tales como arte, deporte, literatura, danza, teatro (entre otras), sin embargo, durante la formación universitaria se verían mermadas por las altas exigencias curriculares. Se pudo inducir que las habilidades innatas y adquiridas en torno a los sentidos y a la imaginación quedaron en alguna medida latentes durante la formación profesional, en especial en cursos superiores. Esta reflexión conlleva a retomar las ideas de Nussbaum (2001) en cuanto al cultivo de la imaginación narrativa de las personas en el plano de la educación a lo largo de la vida. De tal manera que la Educación Superior debería desarrollar en el estudiante la conciencia de la literatura en muchas formas, que le permitan abrir su visión desde una perspectiva global como ciudadano universal con un espíritu deliberante y crítico.

Por otra parte, apareció inusitadamente durante las entrevistas el imaginario de la profesión, que las estudiantes van construyendo a lo largo de sus años de forma- ción universitaria; varias señalaron que se imaginaban como matronas, incluso antes de ingresar a la carrera, otras reconocieron que participar en actividades extracurriculares como comité de extensión, centro de estudiantes y comité de lactancia materna, les permitió acercarse más a la comunidad y de tal manera, sentirse como futuras matronas durante las actividades asistenciales y educativas. De los relatos se pudo inferir que este imaginario les otorgó mayor sentido y motivación a las estudiantes para avanzar, sortear dificultades y lograr alcanzar sus metas.

"Siempre soñé con ser matrona...voy a todas las ferias de salud que puedo porque me gusta mucho el rol de educar así que yo feliz yendo para allá” (E2).

Estos relatos tienen especial significado, porque demostraron que las vivencias universitarias fuera del currículo también empoderan a las personas en sus respectivas disciplinas, y les permitió reforzar capacidades o sellos personales en pro de un ejercicio profesional empático y comprometido.

\subsection{Emocionarse y expresar sentimientos}

La expresión de sentimientos se dió de manera espontánea durante las entrevistas, a través de diversas actitudes expresadas verbalmente y/o gestualmente, que reflejaron alegría, satisfacción, orgullo, y también en algunos casos sentimientos de ansiedad, estrés o frustración frente a algunas situaciones. Para comenzar, todas las estudiantes coincidieron en reconocer que esta capacidad la han forjado a lo largo de sus vidas y según sus experiencias previas, en el seno de su núcleo familiar y con sus amistades. Llama poderosamente la atención que gran parte de las estudiantes manifestaron sentirse felices de haber elegido la carrera, lo que demostró por un lado que esta capacidad la han desarrollado y pudieron expresarla conscientemente, también develó un alto grado de motivación e interés por la disciplina escogida.

"He tenido una muy buena experiencia acá en la uni- 
versidad, me he sentido súper acogida, creo que he crecido como persona, me siento más capaz..." (E4).

En este sentido, se reafirma la tesis de Nussbaum (2005) la cual afirma que el juicio acerca de lo que puede considerarse como valioso o como carente de valor no se sigue de axiomas o principios hieráticos, ya que siempre se valora a partir de patrones humanos. El valor es antropocéntrico, no establecido de forma completamente independiente de los deseos y necesidades de los seres humanos; pero afirmar esto, está muy lejos de afirmar que cada preferencia de cada ser humano cuenta a efectos evaluativos. Los valores son plurales, se refieren a la estimación positiva de todo aquello que le da dignidad a la existencia y que, por tanto, se torna fundamental para buscar una vida feliz.

El sentimiento de felicidad en torno a su formación profesional, expresado por un amplio grupo de estudiantes entrevistadas, es un resultado relevante para la investigación. Según la tesis cognitivista de Nussbaum, queda de manifiesto que las creencias son una condición necesaria y/o suficiente de las emociones y que estas pueden ser aprehendidas a lo largo de la vida. De tal manera, que este resultado abrió una gran puerta para que la carrera trabaje las emociones desde los primeros años de formación, mediante diversas estrategias como programas de apoyo y seguimiento estudiantil, programas de tutorías y acompañamiento, también mediante la incorporación de actividades lúdicas y talleres recreativos que faciliten la expresión de sentimientos y emociones; orientándolas hacia el bienestar en general, lo que de alguna forma se verá reflejado a lo largo de la carrera y en sus propias vidas, mediante un actuar empático, respetuoso y sensible a las necesidades biopsicosociales propias y de las demás personas.

\section{CONCLUSIONES}

Esta investigación en torno al desarrollo de la capacidad de razón práctica durante la vida universitaria, facilitó que se interconecten un sin fin de relatos y vivencias que en concordancia con lo expuesto por Nussbaum en su EDC, permitieron plantear que los estudios universitarios constituyeron esferas de oportunidades que rodearon y envolvieron a las estudiantes de manera dinámica y permeable, en donde las vivencias de nuevas experiencias formativas les permitió cultivar y potenciar gran parte de sus capacidades, en especial la razón práctica; otorgándoles oportunidades para acrecentarlas y consolidarlas para que estas permanezcan y puedan proyectarlas a lo largo de sus vidas. Asimismo, los estudios universitarios abrieron nuevas oportunidades ligadas a mejorar la calidad de vida de las personas y sus familias, profundizando en el pensamiento crítico y práctico, vinculándolas con su propia identidad, a partir de prácticas sociales que promovieron el respeto por el otro desde una mirada inclusiva, ética y biopsicosocial. Cabe destacar que, la prospectiva ofrecida en este estudio se sustentó en los propios relatos de las entrevistadas, en torno a promover un enfoque educacional basado en las ideas de capacidades humanas de Nussbaum, la cual brinda una sólida base para repensar los modelos educativos centrados en el estudiante como fin en sí mismos y en la potenciación de sus habilidades y talentos para llevar vidas universitarias plenamente libres y justas.

Por otra parte, en este trabajo investigativo quedó de manifiesto que el espacio universitario proporciona a las personas del sexo femenino la posibilidad de romper temas de desigualdad de género, inequidad de oportunidades, pobreza, marginalidad, falta de dignidad o valoración de sí mismas. Sin duda en la actualidad las teorías más abstractas o filosóficas están influyendo en materia educativa y social a nivel nacional e internacional, en torno a retomar conceptos basados en el ser humano y en sus derechos. Por lo cual se hace esencial introducir este enfoque en las políticas públicas sobre educación a lo largo de la vida, incorporarlos en las prácticas educativas, alejando de esta forma el modelo utilitarista y profesionalista imperante aún en Chile y en algunos países de Latinoamérica. Por último, esta investigación y la posibilidad cierta de deliberar públicamente sobre ésta y otras teorías relacionadas son un camino propicio para avanzar hacia el respeto 
a los derechos de las personas, en especial al estar insertas en un entorno universitario que juega un rol garante en la potenciación de sus capacidades humanas.

\section{Referencias bibliográficas}

Aristizábal, A. B. (2012). Las capacidades para el desarrollo humano. Cuadernos de Pedagogía, (422), 92-95.

Arnal, J. (1992). Investigación educativa. En metodologías de investigación educativa. España: Labor.

Arjona, G. E. (2013). Democracia y liberalismo político. La perspectiva de Martha Nussbaum. Colombia Internacional, (78), 145-180.

Cohen, M. Z., y Omery, A. (2005). Capítulo 8: Escuelas de fenomenología: implicaciones para la investigación. Asuntos Críticos en Los Métodos de Investigación Cualitativa, 185-207.

Colmenarejo, R. (2016). Enfoque de capacidades y sostenibilidad, aportaciones de Amartya Sen y Martha Nussbaum. Ideas y Valores, 65(160), 121-149.

Fonnegra, C. P. (2013). Martha Nussbaum: la relación entre literatura y filosofía desde una perspectiva aristotélica. Katharsis, (16), 245-265.

Gómez, A. (2013). Ética del desarrollo humano según el Enfoque de Capacidades de Martha Nussbaum. Phainomenon, (12), 19-28.

González, R. (2014). Martha Nussbaum: Humanidades y Universidad en el siglo XXI. Estudios: Filosofía, Historia, Letras, (110), 69-104.

Leal, C. (2012). La noción de justicia en Martha Nussbaum. Moralia, 35(3):407-434.

Monereo, C. (2015). Martha C. Nussbaum-Otro Enfoque para la defensa del ser humano y de los derechos de las mujeres, 36(70), 93-114.

Novales-Alquézar, A. (2016). Ciudadanía y emociones: Hacia una verdadera interculturalidad (a propósito de la teoría cognitiva de la emoción de Martha Nussbaum). Díkaion, 25(1), 12-52.

Nussbaum, M.C. (1998). Virtudes no relativas, un en- foque aristotélico. La calidad de vida. México: Fondo de Cultura Económica.

Nussbaum, M.C. (2001). El cultivo de la humanidad. Una defensa clásica de la reforma en la educación liberal. España: Paidós.

Nussbaum, M.C. (2005). El conocimiento del amor. Ensayos sobre filosofía y literatura. España: Antonio Machado Libros.

Nussbaum, M.C. (2010). Sin fines de lucro. Por qué la democracia necesita de las humanidades. España: Katz editores.

Nussbaum, M.C. (2012a). Crear capacidades: Propuesta para el desarrollo humano. Barcelona: Paidós.

Nussbaum, M.C. (2012b). Las mujeres y el desarrollo humano. El enfoque de las capacidades. Barcelona: Herder.

Prudencio, J. J. B. (2010). La ciudadanía cosmopolita de Martha Nussbaum. Daimon Revista Internacional de Filosofía, 0(0), 347-354.

Ruiz Olabuénaga, J.I. (2011). Metodología de la investigación cualitativa (5a ed.), Universidad de Deusto. Bilbao, España.

Sandín, M.P. (2003). Investigación Cualitativa en Educación. Fundamentos y Tradiciones. Mc Graw and Hill Interamericana de España. Madrid, España: Mc Graw and Hill Interamericana de España.

Schütz, Alfred. El problema de la realidad social. Mauricio Natanson compilador. Buenos Aires: Amorrortu, 1995.

Spradley, J.P. (1979). La entrevista etnográfica. Nueva York: Holt, Rinehart \&Winston.

Tójar, J.C. y Serrano, J. (2000). Ética e investigación educativa. Revista electrónica de investigación y evaluación Educativa, 6, (2) 2-2.

Vilafranca, I., y Buxarrais, M. R. (2009). La educación para la ciudadanía en clave cosmopolita. La propuesta de Martha Nussbaum. Revista Española de Pedagogía, (242), 115-130. 